Mathematical Modelling and Analysis

Volume 18 Number 2, April 2013, 236-249

http://dx.doi.org/10.3846/13926292.2013.781070

(c) Vilnius Gediminas Technical University, 2013
Publisher: Taylor\&Francis and VGTU

http://www.tandfonline.com/TMMA

Print ISSN: 1392-6292

Online ISSN: 1648-3510

\title{
Mathematical Models to Estimate the Mass of Leaf and Sketch the Shape of Tree
}

\author{
Jun $\mathbf{W u}^{a}$ and Yicheng Liu ${ }^{b}$ \\ ${ }^{a}$ Changsha University of Science Technology \\ 410114 Changsha, China \\ ${ }^{b}$ National University of Defense Technology \\ 410073 Changsha, China \\ E-mail(corresp.): junwmath@hotmail.com \\ E-mail: liuyc2001@hotmail.com
}

Received May 10, 2012; revised January 25, 2013; published online April 1, 2013

\begin{abstract}
The mass of leaf is a key factor to estimate the magnitude of biogenic hydrocarbon emission. In this paper, following the minimum material consumption assumption and the conservation law of energy, we build a mathematical model to calculate the mass of leaf, crown and the whole tree, respectively. Also, we translate the shape of crown to match the solution of a second order differential equations with boundary value conditions. Meanwhile, we try to explore what does the climatic zone affect the shape and thickness of leaf. In the simulation section, by using the measured data for 14 trees in 3 different species, we present various simulation results through our models and formulas. Finally, following our models and formulas, we find out some hidden relationships: 1 . There is an intrinsic link between the single leaf area and the hardness of the stem; 2. There is an interconnected relationship between the shape of leaf and the shape of tree; 3 . There are lots of trees with large and thick leaves living in the torrid zone and few in the cold zone.
\end{abstract}

Keywords: leaf mass, tree shape, mathematical model, conservation law of mass, differential equation.

AMS Subject Classification: 93A30; 97M99; 34M99.

\section{Introduction}

With the evolution over several thousand years, each part of the tree has formed a fully functional and interrelated unity for adapting to the external environment and absorbing enough nutrient to meet its regular growth. For example, the size of outside surface of crown should guarantee the balance between the goals to catch enough sunlight and nutrients for the growth and to increase the weight. It is a natural question what is the ideal crown's surface size. Similarly, is there a hidden relationship between the tree shape and the leaf shape? Why do the most broad-leaved trees always live in the tropical zone, coniferous trees 
are rare in tropical regions? After long-term research and exploration, there has been found the quantitative relationship between leaf area and foliage quality $[12,13]$. Recently, authors in $[14,17]$ found a hidden relationship between the vein and the environment. Also, Alexandra [1], Brodribb [3,4], Karlik and Winer [8] and Li et al. [10] try to measure or estimate the leaf mass for some special tree species. More literatures on the topic of tree shape and leaf mass can be seen in $[2,5,6,7,9,16]$ and references therein.

Inspired by the previous work, in this paper, we try to build a mathematical model to find a solution to estimate the leaf mass and sketch the tree shape by the conservation principle of mass and energy. Meanwhile, following on our models and formulas, we find some non-obvious relationships:

1. There is a hidden relationship between the single leaf area and the hardness of the stem.

2. There is an interconnected relationship between the shape of leaf and the shape of tree.

3. There are lots of trees with large and thick leaves living in the torrid zone and few in the cold zone.

To the brief statement, we assume that: (1) A tree shape is axis-symmetric and the leaves have the same size (in an average sense) of thickness and area in one species; (2) The tree stem fits the minimum material consumption assumption: the change of stem weight is just equal to the product of the changes of cross-sectional stem area and the maximum pressure at the cross-sectional plane. (3) Some environment factors and the internal structure of the crown, such as branches numbers, insertion angles, are ignored.

\section{Model Formulation}

Assume that the tree is vertical with ground and the cross-sectional area of the stem is $\left(S(h), m^{2}\right)$ at the height $(h, m)$, where the second term is the unit of the first variable. That is, the unit of cross-sectional area $S(h)$ is $m^{2}$ (square meter). Let the density of the stem be a constant $\left(\rho, \mathrm{kg} \cdot \mathrm{m}^{-3}\right)$ and the vertical intensity of pressure for the stem be $\left(P(h), N \cdot m^{-2}\right)$. When the height of stem increases from $h$ to $h+\Delta h$, the cross-sectional area will decrease from $S(h)$ to $S(h+\Delta h)$. According to the minimum material consumption assumption, the change of weight $\Delta G$ should be properly equal to the product of the changes of cross-sectional area $\Delta S$ and the pressure $P(h)$. Mathematically, we have $\Delta G=-\Delta S \cdot P(h)$, where the minus sign means that the area is decreasing on the variable $h$. Also, by direct computation, we have that $\Delta G \simeq S(h) \cdot \Delta h \cdot \rho \cdot g$, where $g$ is the acceleration of gravity. From above arguments, we see that

$$
\frac{\Delta S}{\Delta h}=-\frac{S(h) \rho g}{P(h)} .
$$

Let $\Delta h$ go to zero, we conclude that

$$
\frac{d S}{d h}=-\frac{S(h) \rho g}{P(h)} .
$$


Denoting the cross-sectional area on floor as $S_{0}$, that is $S(0)=S_{0}$, then we obtain the following Cauchy problem which describes the relationship between cross-sectional area and the height of the stem

$$
\frac{d S}{d h}=-\frac{S(h) \rho g}{P(h)}, \quad S(0)=S_{0} .
$$

Solving it, we get that

$$
S(h)=S_{0} e^{-\rho g \int_{0}^{h} \frac{1}{P(x)} d x} .
$$

In order to estimate the total mass of tree, we assume the mass of branch and leaf concentrate to the stem, and then the height of tree is sufficiently high. Thus the total mass of the tree is given as

$$
M=\rho \int_{0}^{+\infty} S(h) d h=\rho S_{0} \int_{0}^{+\infty} e^{-\rho g \int_{0}^{h} \frac{1}{P(x)} d x} d h .
$$

\subsection{Model simplification}

For the simplification, we assume that $P(h)=P_{0}-r h$, where $P_{0}$ denotes the vertical pressure for the stem at the bottom and $r$ is a constant. Since the pressure is nonnegative, we can estimate approximately the maximum tree height $H^{*}$, which would be $\frac{P_{0}}{r}$.

In this case, we rewrite the Cauchy problem (2.1) as

$$
\frac{d S}{S}=-\frac{\rho g}{P_{0}-r h} d h, \quad S(0)=S_{0}
$$

Integrating from 0 to $h$, we have

$$
\left.\ln (S(x))\right|_{0} ^{h}=\left.\frac{\rho g}{r} \ln \left(P_{0}-r x\right)\right|_{0} ^{h}
$$

Thus

$$
S(h)=S_{0}\left(1-\frac{r}{P_{0}} h\right)^{\frac{\rho g}{r}}
$$

Then the total mass of the tree should be

$$
M=\rho S_{0} \int_{0}^{P_{0} / r}\left(1-\frac{r}{P_{0}} h\right)^{\rho g / r} d h=\frac{\rho S_{0} P_{0}}{\rho g+r} .
$$

At this stage, we should consider a given tree with the height $(H, m)$ and the crown height $\left(H_{c}, m\right)$. From the formulation $(2.2)$, the mass of stem under crown is calculated by $\rho S_{0} \int_{0}^{H-H_{c}}\left(1-\frac{r}{P_{0}} h\right)^{\frac{\rho g}{r}} d h$. Thus the mass of crown should be given as

$$
M_{c}=\rho S_{0} \int_{H-H_{c}}^{P_{0} / r}\left(1-\frac{r}{P_{0}} h\right)^{\rho g / r} d h=\frac{\rho S_{0} P_{0}}{\rho g+r}\left[1-\frac{r}{P_{0}}\left(H-H_{c}\right)\right]^{1+\rho g / r} .
$$


On the other hand, we estimate the mass of crown from the viewpoint of substance exchange. Denote $\left(A_{s}, m^{2}\right)$ as the single leaf area, $(z, m m)$ as the thickness of leaf and $(l, m)$ the perimeter of leaf surface. By the Fick's law $[11,15]$, within a unit time and a unit area, the mass of exchanged substance between a single leaf and outside is described as $m_{e}=D \Delta C / \delta$, where $\Delta C$ denotes the concentration difference inside and outside, $D$ a diffusion coefficient and $\delta$ as the leaf boundary layer thickness, which can be calculated by $\frac{A_{s}}{l}$. Then the total mass of exchanged substance within $\tau$ hours can be formulated by

$$
M_{e}=\frac{A_{\text {total }}}{A_{s}} m_{e} A_{s} \tau=\frac{A_{\text {total }}}{A_{s}} D \Delta C l \tau
$$

where $A_{\text {total }}$ is the total area of tree leaves, which will be formulated later. Meanwhile, the total mass of leaves can be computed as $M_{\text {leaf }}=A_{\text {total }} z \rho_{\text {leaf }}$, where $\rho_{\text {leaf }}$ is the mean density of leaf. Thus $A_{\text {total }}=\frac{M_{\text {leaf }}}{z \rho_{\text {leaf }}}$.

Since the main exchanged substance between leaf and outside is water, and also the crown mass includes water ranged from 70 to 80 percentage, according to the conservation of mass, we set that $M_{e}=Q M_{c}$, where $Q$ denotes the proportion coefficient. Combining the equation (2.3) and (2.4), we have

$$
Q \frac{\rho S_{0} P_{0}}{\rho g+r}\left[1-\frac{r}{P_{0}}\left(H-H_{c}\right)\right]^{1+\frac{\rho g}{r}}=\frac{A_{\text {total }}}{A_{s}} D \Delta C l \tau=\frac{M_{\text {leaf }}}{A_{s} z \rho_{\text {leaf }}} D \Delta C l \tau .
$$

This formula implies that there are some complex relationships among the parameters $S_{0}, A_{\text {total }}, A_{s}, \tau, \rho, l, r, P_{0}$ and $z$. Particularly, we have the following statements.

\section{Conclusions 1}

1. As the other parameters are fixed, with the single leaf area $A_{s}$ increasing, the pressure $P_{0}$ turns more smaller, or the crown height $H_{c}$ goes more lower, or the thickness of leaf $z$ becomes more thinner. Thus there is a hidden relationship between the single leaf area and the hardness (dependent on the value of $P_{0}$ ) of the stem.

2. As the other parameters are fixed, there is a reciprocal proportion relationship between the perimeter of leaf surface $l$ and the exchange substance period $\tau$. That is, if the single leaf area is the same, then the species with sawtooth leaf will grow fast ( $\tau$ is small).

3. As the other parameters are fixed, the total leaf area $A_{\text {total }}$ increase if $\tau$ gets smaller. Thus, in order to absorb enough nourishment within the best time, the most species tries their best to expose leaves and minimize overlapping individual shadows.

\subsection{Crown shape model}

Following the above arguments, we conclude that the shape of crown is sketched by maximizing the outside surface with a given crown volume. For the symmetry of crown, we regard the outside surface as a surface of revolution by a 
curve, and its parameter equation is given by

$$
x(t)=\varphi(t), \quad z(t)=\phi(t), \quad t \in[\alpha, \beta] .
$$

Thus the volume of the crown is computed by

$$
V_{c}=\pi \int_{\alpha}^{\beta} x^{2}(t) z^{\prime}(t) d t=\pi \int_{\alpha}^{\beta} \varphi^{2}(t) \phi^{\prime}(t) d t .
$$

Also, by the computation formula of revolution surface area, we have

$$
A_{c}=2 \pi \int_{\alpha}^{\beta} x(t) \sqrt{x^{\prime 2}(t)+z^{\prime 2}(t)} d t=2 \pi \int_{\alpha}^{\beta} \varphi(t) \sqrt{\varphi^{\prime 2}(t)+\phi^{\prime 2}(t)} d t .
$$

In order to sketch the shape of crown, we introduce the following optimization problem

$$
\operatorname{Maximize}\left\{2 \pi \int_{\alpha}^{\beta} \varphi(t) \sqrt{\varphi^{\prime 2}(t)+\phi^{\prime 2}(t)} d t\right\}
$$

subject to

$$
\left\{\begin{array}{l}
\pi \int_{\alpha}^{\beta} \varphi^{2}(t) \phi^{\prime}(t) d t=V_{c}, \\
\phi(\beta)-\phi(\alpha)=H_{c} .
\end{array}\right.
$$

We introduce a new variable $\lambda$ called a Lagrange multiplier and study the Lagrange function defined by

$$
\begin{aligned}
I & =2 \pi \int_{\alpha}^{\beta} \varphi(t) \sqrt{\varphi^{\prime 2}(t)+\phi^{\prime 2}(t)} d t+\lambda\left(\pi \int_{\alpha}^{\beta} \varphi^{2}(t) \phi^{\prime}(t) d t-V_{c}\right) \\
& =2 \pi \int_{\alpha}^{\beta} L\left(\varphi, \varphi^{\prime}, \phi, \phi^{\prime}, \lambda\right) d t .
\end{aligned}
$$

Then the corresponding $E-L$ equations are shown as follows:

$$
\left\{\begin{array}{l}
\frac{\partial L}{\partial \varphi}=\frac{d}{d t}\left(L_{\varphi^{\prime}}\right) \\
\frac{\partial L}{\partial \phi}=\frac{d}{d t}\left(L_{\phi^{\prime}}\right) \\
\frac{\partial L}{\partial \lambda}=0
\end{array}\right.
$$

Simplifying it, we have

$$
\left\{\begin{array}{l}
\sqrt{\varphi^{\prime 2}+\phi^{\prime 2}}+\lambda \varphi \varphi^{\prime} \phi^{\prime}=\frac{1}{\sqrt{\varphi^{\prime 2}+\phi^{\prime 2}}}\left[\varphi^{\prime 2}+\varphi \varphi^{\prime \prime}-\frac{\varphi \varphi^{\prime}}{\varphi^{\prime 2}+\phi^{\prime 2}}\left(\varphi^{\prime} \varphi^{\prime \prime}+\phi^{\prime} \phi^{\prime \prime}\right)\right] \\
\lambda \varphi \varphi^{\prime}+\frac{1}{\sqrt{\varphi^{\prime 2}+\phi^{\prime 2}}}\left[\varphi^{\prime} \phi^{\prime}+\varphi \phi^{\prime \prime}-\frac{\varphi \phi^{\prime}}{\varphi^{\prime 2}+\phi^{\prime 2}}\left(\varphi^{\prime} \varphi^{\prime \prime}+\phi^{\prime} \phi^{\prime \prime}\right)\right]=0 \\
\varphi^{2} \phi^{\prime}=\frac{H_{c}}{\pi} .
\end{array}\right.
$$


Also, it should subject to the boundary conditions

$$
\varphi(\alpha)=\alpha_{0} ; \quad \varphi(\beta)=\beta_{0} ; \quad \phi(\alpha)=\alpha_{1}, \quad \phi(\beta)=\beta_{1} .
$$

Thus, the shape of crown should be sketched by the solution of this differential equations with boundary value conditions. However, it is difficult to simplify the above boundary value problem for achieving more details about the crown shape. So, at the next step, we investigate the crown shape with two given cases.

\subsubsection{Heart-like curve case}

At first, we consider the anti-heart like curve given by the polar coordinate equation

$$
\rho(\theta)=a(b+\sin (\theta)), \quad \text { where } a, b \text { are parameters, and } \theta \in\left[-\frac{\pi}{2}, \frac{\pi}{2}\right] .
$$

Thus, with the help of Mathematica Soft, the outside surface area can be shown as follows:

$$
A_{c}=\frac{4 \pi a^{2}}{15}\left(5 b^{3}+\frac{1}{b}+10 b-10\right) .
$$

Also, the volume of crown is given by

$$
V_{c}=\pi a^{3} \frac{2 b^{2}+b^{4}}{3} .
$$

Meanwhile, we see that

$$
\phi\left(\frac{\pi}{2}\right)-\phi\left(-\frac{\pi}{2}\right)=2 a b=H_{c} .
$$

From above two formulas, we can deduce the values of $a$ and $b$ :

$$
a_{0}=\frac{6 V_{c}+\sqrt{\left(6 V_{c}\right)^{2}-\pi^{2} H_{c}^{6}}}{2 \pi H_{c}^{2}}, \quad b_{0}=\frac{24 V_{c}+\sqrt{\left(24 V_{c}\right)^{2}-16 \pi^{2} H_{c}^{6}}}{2 \pi H_{c}^{3}} .
$$

Thus the shape of the crown is determined perfectly by the curve shown as the polar equation,

$$
\rho(\theta)=a_{0}\left(b_{0}+\sin (\theta)\right), \quad \theta \in\left[-\frac{\pi}{2}, \frac{\pi}{2}\right]
$$

The projection area of tree is determined by maximum of the function $\varphi(t)$, which is the projection radius of tree. By direct computation, we see that

$$
\begin{aligned}
W_{\max } & =\max _{\theta \in\left[-\frac{\pi}{2}, \frac{\pi}{2}\right]}\{\varphi(\theta)\}=\max _{\theta \in\left[-\frac{\pi}{2}, \frac{\pi}{2}\right]}\{a(b+\sin (\theta)) \cos (\theta)\} \\
& =\frac{\left(3 a b+a \sqrt{b^{2}+8}\right) \sqrt{4-b^{2}+b \sqrt{b^{2}+8}}}{8 \sqrt{2}} .
\end{aligned}
$$


In fact, the value of $W_{\max }$ can be measured from the objective tree, see the data of the column $w$ in Table 1 .

We assume that the leaves are layered and self-resembled. Denote the layer number as $n_{c}$ and the shape of the $k$-th layer is considered as a surface of revolution created by

$$
\rho(\theta)=a_{k}\left(b_{k}+\sin (\theta)\right), \quad \theta \in\left[-\frac{\pi}{2}, \frac{\pi}{2}\right],
$$

where $a_{k}=a_{0}\left(1-\frac{k}{n_{c}}\right), b_{k}=b_{0}\left(1-\frac{k}{n_{c}}\right)$. So, the area of $k$-th surface is

$$
A_{k c}=\frac{4 \pi a_{k}^{2}}{15}\left(5 b_{k}^{3}+\frac{1}{b_{k}}+10 b_{k}-10\right) .
$$

Thus the total area of tree leaves is shown as

$$
A_{\text {tatal }}=\sum_{k=0}^{n_{c}-1} \frac{\alpha_{k}}{1-\beta_{k}} A_{k c}=\frac{4 \pi}{15} \sum_{k=0}^{n_{c}-1} \frac{\alpha_{k} a_{k}^{2}}{1-\beta_{k}}\left(5 b_{k}^{3}+\frac{1}{b_{k}}+10 b_{k}-10\right),
$$

where $\alpha_{k}$ and $\beta_{k}$ are the cover rate and overlap rate, respectively. Naturally, $A_{0 c}=A_{c}$.

\subsubsection{Ellipse curve case}

In this case, we take the parameter equation of ellipse curve as following

$$
x(t)=R \cos (t), \quad z(t)=H_{c} \sin (t), \quad \text { where } R \text { is parameter and } t \in\left[0, \frac{\pi}{2}\right] .
$$

Thus the volume of crown and outside surface can be formulated as

$$
V_{c}=\frac{2}{3} \pi R^{2} H_{c}, \quad A_{c}=\frac{\pi R^{2}}{2}\left(\sqrt{2}+2\left(\frac{H_{c}}{R}\right)^{2} \frac{\arctan \sqrt{\left(\frac{H_{c}}{R}\right)^{2}-1}}{\sqrt{\left(\frac{H_{c}}{R}\right)^{2}-1}}\right) .
$$

Then, in this case, the shape of crown is sketched by the parameter $R=\sqrt{\frac{3 V_{c}}{2 \pi H_{c}}}$, which is the crown projection radius.

Also, the area of self-resembled $k$-th layer is given as

$$
A_{k c}=\left(1-\frac{k}{n_{c}}\right)^{2} \frac{\pi R^{2}}{2}\left(\sqrt{2}+2\left(\frac{H_{c}}{R}\right)^{2} \frac{\arctan \sqrt{\left(H_{c} / R\right)^{2}-1}}{\sqrt{\left(H_{c} / R\right)^{2}-1}}\right) .
$$

So the total area of leaves is

$$
A_{\text {tatal }}=\sum_{k=0}^{n_{c}-1} \frac{\alpha_{k}}{1-\beta_{k}}\left(1-\frac{k}{n_{c}}\right)^{2} \frac{\pi R^{2}}{2}\left(\sqrt{2}+2\left(\frac{H_{c}}{R}\right)^{2} \frac{\arctan \sqrt{\left(\frac{H_{c}}{R}\right)^{2}-1}}{\sqrt{\left(\frac{H_{c}}{R}\right)^{2}-1}}\right) .
$$




\subsection{Model involving energy}

At this stage, we assume that the mass of the crown $M_{c}$ is distributed uniformly and it does work for the tree to absorb the water from the ground to the crown. Due to the different crown shape, this work to absorb water will be different. For the heart-like curve case, the work is

$$
\begin{aligned}
W_{c}^{h} & =\pi \bar{\rho} \int_{-\frac{\pi}{2}}^{\frac{\pi}{2}} \varphi^{2}(t) \phi^{\prime}(t)\left[H-H_{c}+\phi(t)\right] d t \\
& =\frac{\pi Q M_{c} a^{3} b^{2}}{15 V_{c}}\left[\left(10+5 b^{2}\right)\left(H-H_{c}\right)+a\left(6+5 b^{2}\right)\right],
\end{aligned}
$$

where $\bar{\rho}$ is the average density. For the ellipse curve case, the work is

$$
\begin{aligned}
W_{c}^{e} & =\pi \bar{\rho} \int_{-\frac{\pi}{2}}^{\frac{\pi}{2}} \varphi^{2}(t) \phi^{\prime}(t)\left[H-H_{c}+\phi(t)\right] d t \\
& =\frac{\pi Q M_{c} H_{c} R^{2}}{V_{c}}\left(\frac{2 H}{3}-\frac{5 H_{c}}{12}\right) .
\end{aligned}
$$

On the other hand, in view of the Stefan-Boltzmann law [5], the energy transferred by the photosynthesis process of the leaves per unit time and unit area can be formulated by

$$
J_{\text {leaf }}=\alpha_{\text {leaf }} \sigma T^{4}
$$

where $\alpha_{\text {leaf }}$ is the transfer rate, $\sigma=5.67 \times 10^{-8} \mathrm{~W} \cdot \mathrm{m}^{-2} \mathrm{~K}^{-4}$ and $T$ denotes the temperature. Then, during the period $\tau$, the total energy transferred by a tree is

$$
J_{\text {total }}=\alpha_{\text {leaf }} \sigma T^{4} \tau A_{\text {total }}=\alpha_{\text {leaf }} \sigma T^{4} \tau \frac{M_{\text {leaf }}}{z \rho_{\text {leaf }}} .
$$

Considering the energy conservation law, we achieve a relationship between energy $J_{\text {total }}$ and work $W_{c}$ :

$$
W_{c}=G J_{\text {total }}, \quad \text { where } G \text { is a proportion coefficient. }
$$

For example, as the ellipse curved crown, we have

$$
\frac{\pi Q M_{c} H_{c} R^{2}}{V_{c}}\left(\frac{2 H}{3}-\frac{5 H_{c}}{12}\right)=G \alpha_{\text {leaf }} \sigma T^{4} \tau \frac{M_{\text {leaf }}}{z \rho_{\text {leaf }}} .
$$

This formulation implies that there is an inherent relationship between the thickness of leaf and the climatic zone (associated with temperature $T$ ). When the other parameters are fixed, the thickness of leaf would increase with the temperature increasing. That is the partial reason why the most species with big and thick leaves live at the torrid zone.

\section{Model Analysis}

Without loss the generalization, in this section, we will take the ellipse curved crown as a sample and present the quantitative analysis to the total mass of leaves, the distribution of leaves and the shape of leaf. 


\subsection{Total mass of the leaves}

It follows from (2.10) and $M_{\text {leaf }}=z \rho_{\text {leaf }} A_{\text {total }}$ that the total mass of leaves is given as

$M_{\text {leaf }}=z \rho_{\text {leaf }} \sum_{k=0}^{n_{c}-1} \frac{\alpha_{k}}{1-\beta_{k}}\left(1-\frac{k}{n_{c}}\right)^{2} \frac{\pi R^{2}}{2}\left(\sqrt{2}+2\left(\frac{H_{c}}{R}\right)^{2} \frac{\arctan \sqrt{\left(\frac{H_{c}}{R}\right)^{2}-1}}{\sqrt{\left(\frac{H_{c}}{R}\right)^{2}-1}}\right)$.

By introducing the parameters $c=\sum_{k=0}^{n_{c}-1} \frac{\alpha_{k}}{1-\beta_{k}}\left(1-\frac{k}{n_{c}}\right)^{2}, p=\frac{H_{c}}{R}$ and $A_{p}=$ $\pi R^{2}$, we can rewrite the above formulation as

$$
M_{\text {leaf }}=c z \rho_{\text {leaf }} \frac{A_{p}}{2}\left(\sqrt{2}+2 p^{2} \frac{\arctan \sqrt{p^{2}-1}}{\sqrt{p^{2}-1}}\right) .
$$

Thus, there are several key reasons to determine the total mass of leaves: the projection area $A_{p}$, the proportion $p$ of the height-width of crown, the thickness and density of leaf. Furthermore, the distribution of leaves can be shown by

$$
\frac{M_{\text {leaf }}}{A_{p}}=\rho_{\text {leaf }} \frac{c z}{2}\left(\sqrt{2}+2 p^{2} \frac{\arctan \sqrt{p^{2}-1}}{\sqrt{p^{2}-1}}\right) .
$$

\subsection{Shape of the leaf}

Here, we set $\gamma=\frac{A_{s}}{l}$ to distinguish the difference of leaf for different species. In fact, it would be a large $\gamma$ for the square-like leaf and a small $\gamma$ for the long and thin leaf. Basing on the equation (2.5) and the above formula of total mass of leaves, we have

$$
\frac{A_{s}}{l}=\frac{c D \Delta C \tau A_{p}}{2 Q \rho S_{0} P_{0}}(\rho g+r)\left[1-\frac{r}{P_{0}}\left(H-H_{c}\right)\right]^{-1-\frac{\rho g}{r}}\left(\sqrt{2}+2 p^{2} \frac{\arctan \sqrt{p^{2}-1}}{\sqrt{p^{2}-1}}\right) .
$$

This shows that there is a deep relationship between the shapes of leaf and crown. We will present a further discussion with simulations experiment in the next section.

\section{Simulations}

In this section, we try to calculate the total mass of leaf for three tree species and find the essential relationship between the shape of crown and leaf by simulations. The pictures of example leaves for three tree species are shown at Figure 1-Figure 3.

Now, we should make clear the data in Table 1, which are measured for 14 trees at the Changsha suburb. The data in first two columns denote the perimeters of trunk at around ground and one-meter height, respectively. The values of $H$ and $H_{c}$ are calculated by measuring the length of worker's shadow 

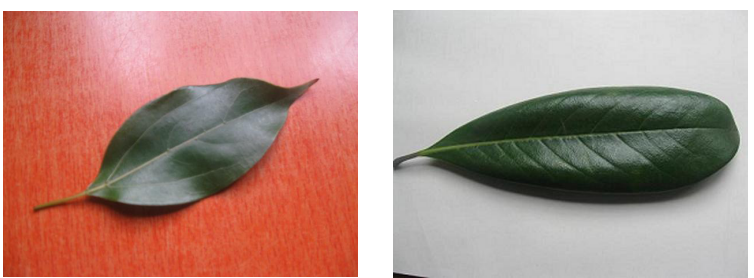

Figure 1. Example leaves (a).

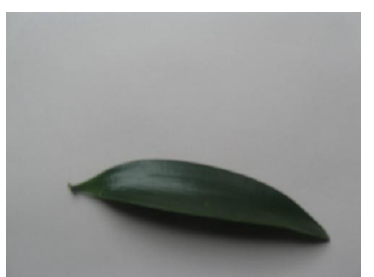

Figure 3. Example leaves (c).

Table 1. The original measured values of parameters.

\begin{tabular}{rllllcccll}
\hline No. & $\pi d_{0}$ & $\pi d$ & $\mathrm{H}$ & $w$ & $A_{s}\left(\times 10^{-3}\right)$ & $H_{c}$ & $\mathrm{z}(\mathrm{mm})$ & $\mathrm{l}$ & Class \\
\hline 1 & 1.26 & 1.03 & 14.05 & 4.14 & 2.5 & 9.81 & 0.031 & 0.2 & $(\mathrm{a})$ \\
2 & 0.51 & 0.46 & 6.5 & 2.64 & 2.3 & 4.41 & 0.029 & 0.2 & $(\mathrm{a})$ \\
3 & 0.54 & 0.47 & 8.8 & 2.8 & 2.3 & 7.2 & 0.029 & 0.2 & $(\mathrm{a})$ \\
4 & 0.6 & 0.51 & 9.4 & 3.05 & 2.3 & 7.31 & 0.029 & 0.2 & $(\mathrm{a})$ \\
5 & 0.58 & 0.5 & 9.12 & 2.96 & 2.3 & 7.22 & 0.029 & 0.2 & $(\mathrm{a})$ \\
6 & 0.84 & 0.7 & 12.04 & 3.21 & 2.4 & 9.4 & 0.03 & 0.2 & $(\mathrm{a})$ \\
7 & 1.1 & 0.9 & 14.84 & 3.51 & 2.5 & 8.42 & 0.031 & 0.2 & $(\mathrm{a})$ \\
8 & 1.03 & 0.88 & 13.25 & 3.28 & 2.5 & 7.9 & 0.031 & 0.2 & $(\mathrm{a})$ \\
9 & 0.97 & 0.75 & 10.41 & 3.53 & 2.5 & 7.81 & 0.031 & 0.2 & $(\mathrm{a})$ \\
10 & 0.86 & 0.71 & 10.73 & 2.91 & 2.4 & 8.91 & 0.03 & 0.2 & (a) \\
11 & 1.07 & 0.87 & 10.3 & 4.5 & 5.7 & 9.3 & 0.041 & 0.46 & (b) \\
12 & 1.32 & 1.02 & 12 & 4.3 & 5.7 & 10.5 & 0.042 & 0.46 & (b) \\
13 & 0.65 & 0.53 & 6.4 & 2.2 & 5.6 & 4.2 & 0.04 & 0.46 & (b) \\
14 & 0.62 & 0.6 & 7 & 2.15 & 0.7 & 5 & 0.08 & 0.18 & (c) \\
\hline
\end{tabular}

and tree's shadow. The value of $w$ is got by measuring the width of vertical projection of crown. The remnant parameters $A_{s}, z$ and $l$ are measured directly.

Also, we will list the input values of the main parameters when simulating this model. The data details can be found in Table 2 .

Table 2. The input values and units of main parameters.

\begin{tabular}{llllllllllll}
\hline Para. & $\rho$ & $n_{c}$ & $\beta$ & $\lambda$ & $T$ & $\tau$ & $\alpha$ & $D$ & $Q$ & $h$ & $\Delta C$ \\
\hline Value & $2.7 \mathrm{E}-4$ & 6 & 0.2 & 0.6 & 260 & 4 & 0.47 & $2.5 \mathrm{E}-5$ & 0.57 & 1.2 & 0.6 \\
Unit & $\mathrm{kg} / \mathrm{cm}^{3}$ & - & - & - & $K$ & $h$ & - & $\mathrm{m}^{3} / \mathrm{s}$ & - & $m$ & - \\
\hline
\end{tabular}

Let $d$ be the ratio of two values $\frac{A_{s}}{l}$ calculated by formula (3.1) and data in Table 1. Then the total mass of leaf $M_{\text {leaf }}(\mathrm{kg})$, the number of leaves $N\left(\times 10^{4}\right)$, the press $P_{0}\left(\times 10^{5} \mathrm{~N} / \mathrm{m}^{2}\right)$, the total mass of tree $M_{\text {total }}$ and the value of $d$ are listed in the Table 3 and Table 4.

It should be pointed out that the mass $M_{\text {total }}$ in Table 3 is the total wet leaf mass. In general, the total dry leaf mass is less than one fifth of $M_{\text {total }}$. Also, the mass $M_{\text {total }}$ in Table 4 is the total wet leaf mass. On the other hand, the value of $d$ at last column is far away the theoretical value 1 . This implies that the ellipsoidal shape of crown doesn't match well the class (c). It is worth 
Table 3. The simulation results for species (a).

\begin{tabular}{lcccccccccc}
\hline Number & 1 & 2 & 3 & 5 & 6 & 7 & 8 & 9 & 10 \\
\hline$M_{\text {total }}$ & 383.7 & 122.5 & 102.2 & 107.8 & 110.25 & 188.4 & 293.8 & 327.9 & 178.6 & 187.9 \\
$M_{\text {leaf }}$ & 74.42 & 20.37 & 34.47 & 38.2 & 36.6 & 53.2 & 54.13 & 47.45 & 50.66 & 45.69 \\
$M_{c}$ & 217.9 & 122.4 & 202.3 & 196.5 & 198.2 & 216 & 131.4 & 132.8 & 209 & 223.3 \\
$\mathrm{~N}$ & 1.00 & 0.32 & 0.54 & 0.60 & 0.57 & 0.77 & 0.73 & 0.64 & 0.68 & 0.66 \\
$P_{0}$ & 0.43 & 0.85 & 0.63 & 0.54 & 0.59 & 0.48 & 0.44 & 0.56 & 0.34 & 0.46 \\
$d$ & 0.94 & 0.72 & 1.11 & 1.04 & 1.06 & 1.22 & 0.95 & 0.96 & 0.88 & 1.27 \\
\hline
\end{tabular}

Table 4. The simulation results for species (b) and (c).

\begin{tabular}{lrrrr}
\hline Number & \multicolumn{1}{l}{11} & \multicolumn{1}{l}{12} & \multicolumn{1}{c}{13} & \multicolumn{1}{c}{14} \\
\hline$M_{\text {total }}$ & 269.61 & 329.94 & 100.86 & 569.27 \\
$M_{\text {leaf }}$ & 102.05 & 111.98 & 22.08 & 50.87 \\
$M_{c}$ & 385.46 & 354.90 & 99.42 & 117.46 \\
$\mathrm{~N}$ & 0.45 & 0.48 & 0.10 & 0.94 \\
$P_{0}$ & 0.42 & 0.34 & 0.43 & 2.69 \\
$d$ & 0.83 & 0.98 & 0.78 & 2.99 \\
\hline
\end{tabular}

to exploring the other shapes to match its crown.

\section{Remarks}

1. Comparing the tree 4 and 14 or 5 and 14, we see that the single leaf area is a sensitivity parameter to estimate the total mass of leaves. Generally, for the trees with the same size of leaf mass and height, the one with large leaf area has lower weight and vice versa.

2. There is an inversely-proportional relationship between the value of pressure $P_{0}$ and the leaf area $A_{s}$. Thus, in most cases, the high hardness (depends on $P_{0}$ ) trees have small single leaf area and the low hardness ones have large single leaf area, see Figure 4.

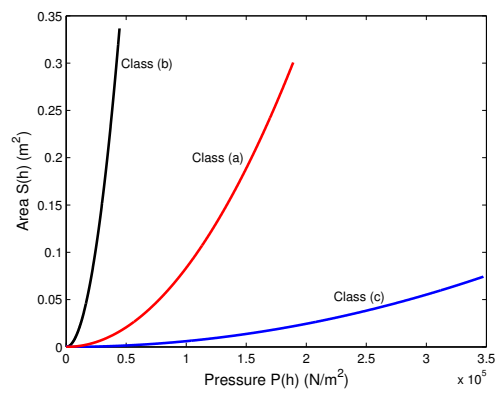

Figure 4. The relationship of pressure and cross-sectional area for species (a),(b) and (c). From these simulation results, we see that the maximum of the intensity of pressure for unit area is class (c), then class (a) and (b) is minimum. Thus there is a hidden relationship between the hardness and the leaf area. 
3. The values of $\frac{A_{s}}{l}$ calculated by formula (3.1) are strongly identical with the values of $\frac{A_{s}}{l}$ calculated by measured data (Table 1 ). The mean value of $d$ is $\bar{d}_{a}=1.019$ in class (a) and the mean value is $\bar{d}=0.985$ in both (a) and (b). Thus, in the average sense, the formula (3.1) describes the shape of leaf well for the species (a) and (b). See Table 3 and Table 4.

4. We detected the hidden relationship among $A_{s}, H_{c}, R$ and $l$. Following the simulation results, we have achieved two simple formulas in the tree classes (a) and (b):

$$
\begin{aligned}
& A_{s}=5.09 \times 10^{-4} \frac{H_{c} l}{R} \quad \text { for class (a); } \\
& A_{s}=4.38 \times 10^{-4} \frac{H_{c} l}{R} \quad \text { for class (b). }
\end{aligned}
$$

Thus there is an interconnected relationship between the shape of leaf $\left(\frac{A_{s}}{l}\right)$ and the shape of tree $\left(\frac{H_{c}}{R}\right)$ in classes (a) and (b), see Figure 5 .

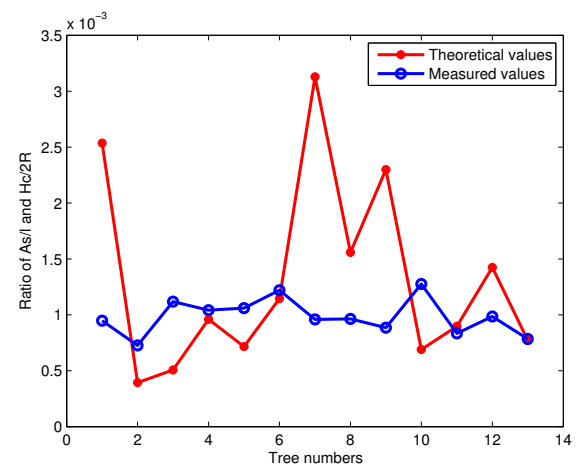

Figure 5. Comparison of theoretical values and measured values for the ratio of $A_{s} / l$ and $H_{c} / 2 R$. The star points (red) and hollow circles (blue) sketch the theoretical values and measured values for the ratio of $A_{s} / l$ and $H_{c} / 2 R$, respectively.

\section{Conclusions}

In this paper, following the minimum material consumption assumption and the conservation law of mass and energy, we formulated a differential equation model to estimate the mass of several parts of tree, which includes the leaf, the crown and the whole tree. Also, we discussed the shape of crown by maximizing the exposed leaves and minimizing the overlapping individual shadows. Furthermore, the shape of crown is associated with the solution of an optimization problem, which finally subjected to a boundary value problem, see as in (2.8). In order to get more details about the hidden relationship between the shape of tree and the shape of leaf, we investigated two cases of the crown shape: the heart-like curve case and the ellipse curve case. Meanwhile, we explored how the climatic zone (temperature) affects the shape and thickness of leaf generally. To certificate our model with the real data, we measured the main 
parameters of 14 trees belonging to 3 tree species. Various simulation results show that our models are identical to the measured data. Finally, following our models and formulas, we figure out some non-obvious relationships:

1. There is a hidden relationship between the single leaf area and the hardness of the stem, see the formula (2.5);

2. There is an interconnected relationship between the shape of leaf and the shape of tree;

3. There are lots of trees with large and thick leaves living in the torrid zone and few in the cold zone, see the formula (2.11).

\section{Acknowledgments}

The authors would like to thank the reviewers for their valuable comments and suggestions. This work was partly supported by Hunan Provincial Education Department Foundation (12B007) and Hunan Provincial Natural Science Foundation of China (11JJ4007).

\section{References}

[1] J. Alexandra and A. Jean-Michel. Variations in leaf mass per area according to $\mathrm{N}$ nutrition, plant age, and leaf position reflect ontogenetic plasticity in winter oilseed rape. Field Crops Research, 114(2):188-197, 2009. http://dx.doi.org/10.1016/j.fcr.2009.07.015.

[2] M.T. Benjamin and A.M. Winer. Estimating the ozone-forming potential of urban trees and shrubs. Atmospheric Environment, 32(1):53-68, 1998. http://dx.doi.org/10.1016/S1352-2310(97)00176-3.

[3] T.J. Brodribb and T.S. Feild. Leaf hydraulic evolution led a surge in leaf photosynthetic capacity during early angiosperm diversification. Ecol. Lett., 13(2):175-183, 2010. http://dx.doi.org/10.1111/j.1461-0248.2009.01410.x.

[4] T.J. Brodribb, T.S. Feild and L. Sack. Viewing leaf structure and evolution from a hydraulic perspective. Funct. Plant. Biol., 37:488-498, 2010. http://dx.doi.org/10.1071/FP10010.

[5] H.A. Cole George and M.M. Woolfson. Planetary Science: The Science of Planets Around Stars (1st ed.). Institute of Physics Publishing, 2002.

[6] V. Dhaval, M. Nirav, J. Dinakaran and N.S.R. Krishnayya. Allometric equations for estimating leaf area index (lai) of two important tropical species. J. Forestry Research, 21(2):197-200, 2010. http://dx.doi.org/10.1007/s11676-010-0032-0.

[7] F. Gianni and S. Ivan. Using leaf mass per area as predictor of light interception and absorption in crop/weed monoculture or mixed stands. Agricultural and Forest Meteorology, 151(5):575-584, 2011. http://dx.doi.org/10.1016/j.agrformet.2011.01.004.

[8] J.F. Karlik and A.M. Winer. Comparison of calculated and measured leaf masses of urban trees. Ecological Appl., 9(4):1168-1176, 1999. http://dx.doi.org/10.1890/1051-0761(1999)009[1168:COCAML]2.0.CO;2. 
[9] J.A. Kershaw and D.A. Maguire. Crown structure in western hemlock, Douglasfir and grand fir in western Washington: Trends in branch-level mass and leaf area. Canad. J. Forest Research, 25:1897-1912, 1995.

http://dx.doi.org/10.1139/x95-206.

[10] B. Li, M. Li, X. Zhou and L. Zhang. Hyperspectral estimation models for nitrogen contents of apple leaves. J. Remote Sensing, 14(4):537-542, 2010.

[11] P. Nobel. Physicochemical and Environmental Plant Physiology. Academic Press, London, 2009.

[12] M.L. Roderick. On the use of thermodynamic methods to describe water relations in plants and soil. Australian J. Plant Physiology, 28(8):729-742, 2001.

[13] M.L. Roderick. On the conservative nature of the leaf mass-area relationship. Ann. Botany, 89(5):537-542, 2002. http://dx.doi.org/10.1093/aob/mcf100.

[14] D.L. Royer and P. Wilf. Why do toothed leaves correlate with cold climates? Gas exchange at leaf margins provides new insights into a classic paleotemperature proxy. Int. J. Plant Sci., 167(1):11-18, 2006. http://dx.doi.org/10.1086/497995.

[15] W.F. Smith. Foundations of Materials Science and Engineering (3rd ed.). McGraw-Hill, 2004.

[16] E.T.F. Witkowski and B.B. Lamont. Leaf specific mass confounds leaf density and thickness. Oecologia, 88:486-493, 1991.

[17] Y. Zhu, H. Kang, Q. Xi, S.Y. Wang and Ch. Liu. Pattern of leaf vein density and climate relationship of Quercus variabilis populations remains unchanged with environmental changes. Trees-Structure and Function, pp. 1-11, 2011. http://dx.doi.org/10.1007/s00468-011-0624-0. 